\title{
Effects of Soil Applications of Micro-Nutrients and Chelating Agent Citric Acid on Mineral Nutrients in Soybean Seeds
}

\author{
Mudlagiri B. Goli1 ${ }^{*}$, Manju Pande ${ }^{1}$, Nacer Bellaloui², Daniele De Wrachien ${ }^{3}$ \\ ${ }^{1}$ Department of Natural Sciences and Environmental Health, Mississippi Valley State University, Itta Bena, \\ MS, USA \\ ${ }^{2}$ USDA, ARS, Crop Genetics Research Unit, Stoneville, MS, USA \\ ${ }^{3}$ Department of Agricultural Engineering, State University of Milano, Milano, Italy \\ Email: "goli@mvsu.edu
}

Received 27 October 2015; accepted 23 November 2015; published 27 November 2015

Copyright (C) 2015 by authors and Scientific Research Publishing Inc.

This work is licensed under the Creative Commons Attribution International License (CC BY).

http://creativecommons.org/licenses/by/4.0/

(c) (i) Open Access

\section{Abstract}

Micro-nutrient deficiency in soil results in crop yield loss and poor seed quality. Correcting this deficiency is normally done by foliar or soil application. The objective of this research was to determine the effects of soil applications of five micro-nutrients $\mathrm{(Mn}, \mathrm{Cu}, \mathrm{Zn}, \mathrm{Mo}$, and $\mathrm{B}$ ) alone and in combination with a chelating agent citric acid (CA) on soybean leaf and seed nutrients. Source of micro-nutrient compounds were $\mathrm{MnCl}_{2}, \mathrm{CuCl}_{2}, \mathrm{ZnCl}_{2}, \mathrm{MoO}_{3}$, and $\mathrm{H}_{3} \mathrm{BO}_{3}$. Our hypothesis was that micro-and macro-nutrients may be transported to leaves and then to seeds at different rates. They may interact synergistically or competitively during the uptake process. A greenhouse experiment was conducted at Mississippi Valley State University, Itta Bena, Mississippi, USA. Soybean cultivar, Bolivar (maturity group V), was applied with micro-nutrients-chelating agent citric acid at V3 and R3 (pod initiation) stage. The results showed that applications of $\mathrm{Cu}, \mathrm{Zn}, \mathrm{B}$ and Mo increased three unrolled trifoliate leaves $\mathrm{Cu}, \mathrm{Zn}, \mathrm{B}$ by $26.5 \%, 13.8 \%, 113 \%$ and $M$ increased to $179 \mathrm{mg} / \mathrm{kg}$, respectively in the leaves. Also, the application of " $\mathrm{Cu}, \mathrm{Zn}, \mathrm{B}$ and Mo increased $\mathrm{Cu}, \mathrm{Zn}, \mathrm{B}$ by $55.5 \%$, $8.2 \%, 28.6 \%$ and Mo increased to $202 \mathrm{mg} / \mathrm{kg}$ " respectively in soybean seeds. Application of Mn had no direct effect on increasing Mn either in leaves or in seeds, however, Mn and Mn + CA treatment affected other mineral contents. Application of $\mathrm{Cu}, \mathrm{Zn}, \mathrm{Mo}$, B and $\mathrm{CA}$ increased macro-nutrients $\mathrm{K}, \mathrm{N}, \mathrm{P}, \mathrm{Mg}$, and $\mathrm{S}$. Irrespective of the applications, the nutrient increase trend in seed was $\mathrm{Na}>\mathrm{Fe}>\mathrm{Zn}>\mathrm{Mn}>\mathrm{B}>\mathrm{Cu}>\mathrm{Mo}$. However, Mo application resulted in the following seed nutrient accumulation pattern: $\mathrm{Na}>\mathrm{Mo}>\mathrm{Fe}>\mathrm{Zn}>\mathrm{Mn}>\mathrm{B}>\mathrm{Cu}$. This may suggest that Mo had higher mobility to seeds than other micro-nutrients. Combination of soil application of Mo + CA increased Mo in leaves at V3 stage; however, Mo + CA soil application during pod-filling stage had no significant effects on Mo accumulation in seeds. The current research showed that some micro-nutrient

${ }^{*}$ Corresponding author. 
application with the chelating agent CA could increase seed nutrients. Since these results are conducted under greenhouse experiments, further research under field conditions is needed before conclusive recommendations are made.

\title{
Keywords
}

\author{
Chealting Agent Citric Acid, Micro-Element Applications, Seed Minerals, Soybean Seeds
}

\section{Introduction}

Many countries in the world depend on soybeans and other legumes as key sources of dietary protein. Soybean is a species of legume native to East Asia, widely grown for its edible bean. The plant is classified as an oilseed rather than a pulse by Agricultural Organizations [1] [2]. Fat-free (defatted) soybean meal is an important cheap source of protein as animal feed and prepackaged meals. Soy vegetable oil is another product of processing the soybean crop. Soybean seeds have the highest protein content amongst other plant products, and contain all eight essential amino acids. Soybeans are processed to various soy products namely soy flour, soy milk, cottage cheese like tofu, fermented products like tempeh and miso [3] [4]. Soybean seeds are also a major source of oil, carbohydrates, minerals, and secondary metabolites such as lignin and isoflavones [5] [6]. It is also reported that soybeans are rich in minerals that are important for human growth and nutrition, for example, $\mathrm{K}, \mathrm{P}, \mathrm{Mg}, \mathrm{Ca}, \mathrm{Fe}$, $\mathrm{Mn}, \mathrm{Zn}$ and $\mathrm{Na}$ [7] [8]. Micro-nutrient deficiency affects more than three billion people of all ages, especially in many developing countries [9]. Plant biofortification to improve mineral concentrations in crops by conventional breeding is regarded as the most economical and effective strategy; however, agronomic intervention, such as application of mineral fertilizer, can be considered as complementary approach to the conventional breeding strategy [10] [11].

In our earlier study it was observed that soil application of $\mathrm{Fe}\left(\mathrm{FeCl}_{2}\right)$ and different chelating agents to soybean increased $\mathrm{Fe}, \mathrm{Cu}, \mathrm{Zn}$, and $\mathrm{B}$ in the seed [12]. In another study, it was reported that application of $\mathrm{Zn}, \mathrm{Mn}$ and B to soil and/or foliar applications altered N, K, P, Mn, Zn and B concentrations in seed [13]. Application of Zn, Mn and B was also reported to increase yield, improving seed quality, and oil [13] [14]. Other studies have also reported that iron-chlorosis symptoms of soybean plants can be reduced by adding a chelating agent with $\mathrm{Fe}$ in Hoagland solutions [15] [16]. Although soil contains all nutrients, under certain conditions, these nutrients, especially the cations, can be tightly bound to the soil and also precipitation can limit their availability to the crop. Chelators can bind to the metals in the soil depending upon competition for a particular metal ions, and can aid the nutrient uptake, transport from leaves (source) to seed (sink), resulting in higher accumulation in seeds. The objective of this study was to investigate if the application of micro-nutrients ( $\mathrm{Mn}, \mathrm{Cu}, \mathrm{Zn}, \mathrm{Mo}$, and $\mathrm{B}$ ) to soil with a natural chelating agent Citric acid (CA) would alter mineral accumulation in seeds. Our hypothesis was that the application of micro-nutrients $\mathrm{Mn}, \mathrm{Cu}, \mathrm{Zn}, \mathrm{Mo}, \mathrm{B}$ alone or with the chelating agent citric acid would influence the accumulation of minerals in leaves, and consequently result in altering seed mineral composition.

\section{Materials and Methods}

A greenhouse experiment was conducted at Mississippi Valley State University, Itta Bena, Mississippi, USA (latitude of N $33^{\circ} 28^{\prime}$ and longitude W $90^{\circ} 20^{\prime}$ ). Soybean cultivar Bolivar (maturity group V) was planted in 48 pots (4 gallon). Pots were arranged in a randomized complete block design. The planting of six seeds in each pot was done in early June 2013. Four replicates were used for each treatment. The top soil used for this experiment was analyzed for mineral concentrations (mg/kg) were: $\mathrm{Na}=41.7, \mathrm{Mn}=204.6, \mathrm{Cu}=0.5, \mathrm{Fe}=4185, \mathrm{Zn}=27.5$, $\mathrm{Mo}=0.5, \mathrm{~B}=2.6$. The macro-nutrients were $\mathrm{K}=1,469, \mathrm{Ca}=4,491, \mathrm{Mg}=838, \mathrm{P}=257, \mathrm{~S}=427$. $\mathrm{N}$ wasnot analysed by the analytical lab. Ten days after germination, soybean seedlings were watered twice a week.

To prepare the solution, $2.4 \mathrm{mmol}$ of the compounds, $\mathrm{MnCl}_{2}, \mathrm{CuCl}_{2}, \mathrm{ZnCl}_{2}, \mathrm{MoO}_{3}$, and $\mathrm{H}_{3} \mathrm{BO}_{3}$ were dissolved in $1000 \mathrm{ml}$ of deionized water (DI). Then, $125 \mathrm{ml}(0.30 \mathrm{mmol})$ of the solution were used per pot. The chelating agent CA was prepared by dissolving $7.8 \mathrm{mmol}$ of CA in $2000 \mathrm{ml}$ of DI water. The $76 \mathrm{ml}$ of this CA solution was applied to each pot. The micro-nutrients were applied to pots (soil application) as separate or in combina- 
tion of CA such as Mn (separate) or Mn + CA (in combination).

\subsection{Treatments}

The minerals were applied twice, one week apart, at V3 stage of soybean plants. The pots were watered twice a week. The plants were grown under greenhouse conditions of natural light and temperature. One week after two chemical applications, three plants were picked up from each pot for leaf mineral analysis. In late September, during R3 (pod initiation stage), the plants were subjected to one final chemical application. The other three plants in each pot were grown until maturity ( $3^{\text {rd }}$ week of October). The temperature in the greenhouse throughout this experiment varied from $32.2^{\circ} \mathrm{C}$ to $35^{\circ} \mathrm{C}$. Mature seeds were harvested at R8 (physiological maturity stage) for chemical analyses.

\subsection{Seed, Soil, and Leaf Mineral Analysis}

The top soil results are repored under section 2 under materials and methods. Results from micro- and macromineral analysis of soybean leaves, and soybean seeds are reported in tables [1]-[4]. The anylsis of soil, leaves and seeds were carried out by Soil Water, Plant, and Animal Plant Tissue Analysis Laboratory, University of Georgia, Athens, GA, USA. The collected leaves were dried in oven in brown bags at $65^{\circ} \mathrm{C}$ for $3-5$ days until a constant mass was achieved. The dried leaf samples and seeds were then sent to the lab for minerals analysis. Briefly, for the total elemental analysis (soil and plants), samples were weighed (0.5 g), and $5 \mathrm{ml}$ of concentrated $\mathrm{HNO}_{3}$ was added for microwave digestion (CEM Mars6). The sample and acid (s) were placed in a fluorocarbon polymer (PFA or TFM) microwave vessel. The vessel is sealed and heated in the microwave unit for a specified period of time. After cooling, the vessel contents were filtered, centrifuged, and then diluted to a known volume. The digested solutions were analyzed by Inductively Coupled Plasma-Optical Emission Spectroscopy (ICP-OES) (Spectro Arcos FHS16 AMETEK ICP-OES). All results are reported as parts per million $\left(\mathrm{mg} \cdot \mathrm{kg}^{-1}\right.$ or ug. $\mathrm{g}^{-1}$ ). Further details are available in the reference section as EPA method [17].

\subsection{Experimental Design and Statistical Analyses}

The experiment was a randomized complete block design. Four replicates were used for each treatment. Analysis of variance was conducted using Proc GLM in SAS [18]. Means were separated using Fisher's Least Significant Difference test using $5 \%$ as level of significance.

\section{Results}

\subsection{Leaf Micro- and Macro-Nutrients}

Application of $\mathrm{Cu}, \mathrm{Zn}, \mathrm{B}$ and Mo increased Cu, Zn, B and Mo in leaves by 26.6\%, 13.80\%, 112.7\%, and Mo increased to $179 \mathrm{mg}$ per kg respectively (Table 1). Most of the treatments, except for Mo, decreased Fe by $7.6 \%$ to $30.2 \%$. Mo increased Fe by 5.53\%. $\mathrm{MnCl}_{2}$ application did not change $\mathrm{Mn}$ in leaves or in seeds. Application of CA increased $\mathrm{Mn}$ in leaves by $41.0 \%$. Application of $\mathrm{Mn}, \mathrm{Cu}, \mathrm{Zn}$, Mo and B increased Na concentration in leaves by $211 \%, 141 \%, 50 \%, 190 \%$ and $71 \%$, respectively. Irrespective of treatments, the concentration trend of micro-nutrients was $\mathrm{Na}>\mathrm{Mn}>\mathrm{Fe}>\mathrm{B}>\mathrm{Zn}>\mathrm{Cu}>\mathrm{Mo}$, except for Mo application. Similar trend was seen in our earlier report [19]. In present study the application of Mo resulted in the following concentration trend $\mathrm{Na}>$ $\mathrm{Mo}>\mathrm{Mn}>\mathrm{Fe}>\mathrm{B}>\mathrm{Zn}>\mathrm{Cu}$.

For macro-nutrients in leaves: $\mathrm{Mn}, \mathrm{Cu}, \mathrm{Zn}, \mathrm{B}, \mathrm{CA}$, and $\mathrm{Mn}+\mathrm{CA}$ applications increased $\mathrm{Ca}$ in leaves by 13.2\% to 30.0\% (Table 2), but had no effect on Ca concentration in seeds (Table 4). Mn, Cu and B applications decreased $\mathrm{K}$ in leaves by $7.8,11.6 \%$ and $11.0 \%$, respectively (Table 2). In seeds, B and CA applications increased $\mathrm{K}$ by $7.4 \%$ and $7.9 \%$, respectively (Table 4 ).

\subsection{Seed Micro- and Macro-Nutrients}

Soil applications of $\mathrm{CuCl}_{2}, \mathrm{ZnCl}_{2}$, and $\mathrm{H}_{3} \mathrm{BO}_{3}$ and $\mathrm{MoO}_{3}$, resulted in high $\mathrm{Cu}, \mathrm{Zn}, \mathrm{B}$ and $\mathrm{Mo}$ concentrations in seeds (Table 3). The increases of $\mathrm{Cu}, \mathrm{Zn}, \mathrm{B}$ and $\mathrm{Mo}$ in the seeds were 55.1\%, 8.2\%, 28.6\% and Mo increased to $202 \mathrm{mg}$ per $\mathrm{kg}$ by respective treatments. Mn application did not increase $\mathrm{Mn}$ in seeds. But $\mathrm{Cu}$ and $\mathrm{Zn}$ applications helped increase $\mathrm{Mn}$ in seeds by $28.8 \%$ and $21.1 \%$ respectively. Applications of $\mathrm{MnCl}_{2}, \mathrm{CuCl}_{2}$, and 
Table 1. Leaf micro nutrients concentrations $(\mathrm{mg} / \mathrm{kg})$ as affected by soil micro-nutrient application and chelating agent citric acid (CA).

\begin{tabular}{|c|c|c|c|c|c|c|c|}
\hline Treatments & B & $\mathrm{Cu}$ & $\mathrm{Fe}$ & Mn & $\mathrm{Na}$ & $\mathrm{Zn}$ & ${ }^{* *} \mathrm{Mo}$ \\
\hline $\mathrm{C}$ & 98.7 c, d, e & $5.8 \mathrm{f}$ & $107.7 \mathrm{~b}$ & $104.1 \mathrm{e}, \mathrm{f}$ & $187 \mathrm{~h}$ & $63.8 \mathrm{e}, \mathrm{f} \mathrm{g}$ & 1 \\
\hline T1 (Mn) & $91.6 \mathrm{e}, \mathrm{f}, \mathrm{g}$ & $9.0 \mathrm{~b}(+55.6)$ & $83.7 \mathrm{f}(-22.3)$ & $111 \mathrm{e}, \mathrm{f}$ & $\begin{array}{c}582 \mathrm{a} \\
(+211)\end{array}$ & $61.06 \mathrm{~g}, \mathrm{~h}$ & 2 \\
\hline $\mathrm{T} 2(\mathrm{Cu})$ & 87.2 f, g (-11.2) & 7.3 c (+26.6) & 95.5 d, e (-11.3) & $\begin{array}{c}150 \mathrm{~b} \\
(+43.8)\end{array}$ & $\begin{array}{c}450 \mathrm{~b} \\
(+141)\end{array}$ & $62.3 \mathrm{f}, \mathrm{g}, \mathrm{h}$ & 2 \\
\hline T3 (Zn) & $95.1 \mathrm{~d}, \mathrm{e}, \mathrm{f}$ & $4.42 \mathrm{~g}(-23.7)$ & 90.6 e (-15.9) & $\begin{array}{l}168.0 \mathrm{a} \\
(+61.4)\end{array}$ & $\begin{array}{l}280 \mathrm{~d}, \mathrm{e} \\
(+50.0)\end{array}$ & $\begin{array}{c}72.6 \mathrm{~b} \\
(+13.8)\end{array}$ & 2 \\
\hline T4 (Mo) & 110.6 c (+12.1) & 6.69 c, d, e (+15.5) & 113.6 a $(+5.5)$ & $\begin{array}{c}118.4 \mathrm{c}, \mathrm{d} \\
(+13.68)\end{array}$ & $\begin{array}{c}541 \mathrm{a} \\
(+190)\end{array}$ & $66.1 \mathrm{c}, \mathrm{d}, \mathrm{e}$ & 179 \\
\hline T5 (B) & 209.88 b (+112.7) & $5.78 \mathrm{f}$ & $75.3 \mathrm{~g}(-30.0)$ & $\begin{array}{l}146.1 \mathrm{~b} \\
(+40.3)\end{array}$ & $318 \mathrm{~d}(+71)$ & 64.2 e, d, f, g & 2 \\
\hline T6 (CA) & $86.5 \mathrm{~g}(-12.3)$ & $6.16 \mathrm{~d}, \mathrm{e}, \mathrm{f}$ & 103.5 b, c & $\begin{array}{l}147.3 \mathrm{~b} \\
(+41.5)\end{array}$ & $193 \mathrm{~g}, \mathrm{~h}$ & $65.5 \mathrm{~d}, \mathrm{e}, \mathrm{f}$ & 2 \\
\hline T7 (Mn + CA) & 92.9 d, e, f, g & 9.4 b (+62.4) & $108.7 \mathrm{a}, \mathrm{b}$ & $\begin{array}{c}124.2 \mathrm{c}, \mathrm{d} \\
(+19.3)\end{array}$ & $\begin{array}{c}587 \mathrm{a} \\
(+215)\end{array}$ & 59.0 h (-7.4) & 2 \\
\hline $\mathrm{T} 8(\mathrm{Cu}+\mathrm{CA})$ & 90.8 e, f, g & 10.7 a $(+84.5)$ & 90.2 e $(-16.2)$ & $\begin{array}{c}120.8 \mathrm{c}, \mathrm{d} \\
(+16.0)\end{array}$ & $\begin{array}{c}388 \text { c } \\
(+108)\end{array}$ & $\begin{array}{c}72.5 \mathrm{~b} \\
(+13.80)\end{array}$ & 2 \\
\hline T9 (Zn + CA) & $95.8 \mathrm{~d}, \mathrm{e}$ & $7.00(+21.0)$ & 92.5 b, c (-14.1) & $\begin{array}{c}119.1 \text { c, d } \\
(+14.4)\end{array}$ & $\begin{array}{c}242 \mathrm{e}, \mathrm{f}, \mathrm{g} \\
(+30)\end{array}$ & $\begin{array}{c}78.6 \mathrm{a} \\
(+23.2)\end{array}$ & 3 \\
\hline $\mathrm{T} 10(\mathrm{Mo}+\mathrm{CA})$ & $100.6 \mathrm{~d}$ & 7.3 c $(+25.2)$ & 99.5 c, d (-7.6) & $112.9 \mathrm{~d}, \mathrm{e}$ & $\begin{array}{c}240 \text { e, f, g } \\
(+28.5)\end{array}$ & $65.5 \mathrm{~d}, \mathrm{e}, \mathrm{f}$ & 446 \\
\hline $\mathrm{T} 11$ (B + CA) & 223.5 a (+126.5) & $5.95 \mathrm{e}, \mathrm{f}$ & $83.0 \mathrm{f}(-23)$ & $\begin{array}{l}127.6 \mathrm{c} \\
(+22.6)\end{array}$ & $215 \mathrm{f}, \mathrm{g}, \mathrm{h}$ & $61.5 \mathrm{~g}, \mathrm{~h}$ & 1 \\
\hline
\end{tabular}

*Means given within a column with the same letters are not significantly different at $\mathrm{P}<0.05$. Four replicates were used. The numbers in the brackets are increase $(+)$ or decrease $(-)$ from the control for a given seed nutrient constituent. Source of micro-nutrient compounds used were $\mathrm{MnCl}_{2}$, $\mathrm{CuCl}_{2}$, $\mathrm{ZnCl}_{2}, \mathrm{MoO}_{3}$, and $\mathrm{H}_{3} \mathrm{BO}_{3}$. C = Control; $\mathrm{T}=$ Treatments. ${ }^{* *}$ Mo content was to low (1 - $\left.3 \mathrm{ppm}\right)$ to be compared by SAS for significance value.

Table 2. Leaf macro-nutrient concentrations (\%) in V3 stage leaves as affected by soil micro-nutrient application and chelating agent citric acid (CA).

\begin{tabular}{|c|c|c|c|c|}
\hline Treatments & $\mathrm{Ca}$ & $\mathrm{K}$ & $\mathrm{Mg}$ & $\mathrm{P}$ \\
\hline $\mathrm{C}$ & $0.49 \mathrm{~g}, \mathrm{~h}$ & $3.45 \mathrm{a}$ & $0.31 \mathrm{c}, \mathrm{d}$ & $0.60 \mathrm{c}, \mathrm{d}, \mathrm{e}$ \\
\hline $\mathrm{T} 1(\mathrm{Mn})$ & 0.58 b, c, d (+13.20) & $3.18 \mathrm{c}, \mathrm{d}(-7.8)$ & 0.29 e, f (-6.45) & $0.52 \mathrm{f}(-13.3)$ \\
\hline $\mathrm{T} 2(\mathrm{Cu})$ & 0.56 c, d, e, f (+11.5) & $3.05 \mathrm{~d}$, e $(-11.6)$ & $0.29 \mathrm{f}, \mathrm{g}(-6.5)$ & $0.53 \mathrm{f}(-11.7)$ \\
\hline T3 (Zn) & $0.62 \mathrm{a}, \mathrm{b}(+20.1)$ & 3.39 a, b & $0.35 \mathrm{a}(+12.9)$ & $0.68 \mathrm{a}, \mathrm{b}(+30.8)$ \\
\hline T4 (Mo) & $0.51 \mathrm{e}, \mathrm{f}, \mathrm{g}$ & $3.45 \mathrm{a}$ & $0.3 \mathrm{e}, \mathrm{d}, \mathrm{f}$ & $0.65 \mathrm{a}, \mathrm{b}, \mathrm{c}$ \\
\hline $\mathrm{T} 5$ (B) & $0.61 \mathrm{~b}, \mathrm{c}(+22.2)$ & $3.07 \mathrm{~d}$, e $(-11.0)$ & $0.3 \mathrm{~d}, \mathrm{e}$ & 0.55 e, f \\
\hline T6 (CA) & 0.67 a $(+30.0)$ & 3.02 e $(-12.5)$ & $0.32 \mathrm{~b}(+3.2)$ & $0.52 \mathrm{f}(-13.3)$ \\
\hline $\mathrm{T} 7(\mathrm{Mn}+\mathrm{CA})$ & 0.57 b, c, d, e (+19.1) & $3.13 \mathrm{~d}$, e (-9.3) & $0.27 \mathrm{~h}(-12.9)$ & $0.53 \mathrm{e}, \mathrm{f}$ \\
\hline $\mathrm{T} 8(\mathrm{Cu}+\mathrm{CA})$ & $0.48 \mathrm{~g}, \mathrm{~h}$ & 3.28 b, c $(-4.9)$ & $0.31 \mathrm{c}, \mathrm{d}$ & 0.69 a, b (+15.0) \\
\hline $\mathrm{T} 9(\mathrm{Zn}+\mathrm{CA})$ & $0.48 \mathrm{~g}, \mathrm{~h}$ & $3.10 \mathrm{~d}$, e $(-10.1)$ & $0.28 \mathrm{~g}, \mathrm{~h}(-9.7)$ & 0.69 a, b (+15.0) \\
\hline $\mathrm{T} 10(\mathrm{Mo}+\mathrm{CA})$ & $0.53 \mathrm{~d}, \mathrm{e}, \mathrm{f}, \mathrm{g}$ & $3.38 \mathrm{a}, \mathrm{b}$ & $0.32 \mathrm{~b}, \mathrm{c}$ & $0.57 \mathrm{~d}, \mathrm{e}, \mathrm{f}$ \\
\hline $\mathrm{T} 11(\mathrm{~B}+\mathrm{CA})$ & $0.51 \mathrm{f}, \mathrm{g}$ & $3.05 \mathrm{~d}$, e $(-11.6)$ & 0.29 e, $f(-6.5)$ & $0.64 \mathrm{a}, \mathrm{b}, \mathrm{c}, \mathrm{d}$ \\
\hline
\end{tabular}

*Means given within a column with the same letters are not significantly different at $\mathrm{P}<0.05$. Four replicates were used. The numbers in the brackets are increase (+) or decrease (-) from the control for a given seed nutrient constituent. Source of micro-nutrient compounds used were $\mathrm{MnCl}_{2}$, $\mathrm{CuCl}_{2}$, $\mathrm{ZnCl}_{2}, \mathrm{MoO}_{3}$, and $\mathrm{H}_{3} \mathrm{BO}_{3}$. C = Control; $\mathrm{T}=$ Treatments. 
Table 3. Seed micro-nutrient concentrations $(\mathrm{mg} / \mathrm{kg})$ as affected by soil micro-nutrient application and chelating agent citric acid (CA).

\begin{tabular}{|c|c|c|c|c|c|c|c|}
\hline Treatments & B & $\mathrm{Cu}$ & $\mathrm{Fe}$ & Mn & $\mathrm{Na}$ & $\mathrm{Zn}$ & ${ }^{* *}$ Mo \\
\hline $\mathrm{C}$ & $25.02 \mathrm{c}, \mathrm{d}$ & $8.62 \mathrm{c}, \mathrm{d}$ & 75.98 a, b, c & 54.52 c, d & 1212.7 d, e, f & 63.31 b, c, d & 4 \\
\hline T1 (Mn) & 26.25 b, c, d & 9.21 b, c & $\begin{array}{l}\text { 70.30e, f, g } \\
\quad(-7.5)\end{array}$ & 26.25 b, c, d & 740 h (-39.0) & 59.43 d, e, f & 3 \\
\hline $\mathrm{T} 2(\mathrm{Cu})$ & 26.40 b, c, d & $\begin{array}{l}13.37 \mathrm{a} \\
(+55.1)\end{array}$ & 74.94 b, c, d & $\begin{array}{l}70.24 \mathrm{~b} \\
(+28.8)\end{array}$ & 904 g $(-25)$ & $66.84 \mathrm{a}, \mathrm{b}$ & 3 \\
\hline T3 (Zn) & 24.98 c, d & 9.52 b, c & 79.86 a & $66.0 \mathrm{~b}(+21.1)$ & $1154 \mathrm{f}$ & 68.48 a $(+8.2)$ & 3 \\
\hline T4 (Mo) & 27.1 b, c & 8.93 b, c & 72.32 c, d, e & $58.12 \mathrm{c}$ & 1277 c, d, e & $\begin{array}{c}59.13 \text { e, f } \\
(-6.6)\end{array}$ & 202 \\
\hline T5 (B) & $\begin{array}{l}32.18 \text { a } \\
(+28.6)\end{array}$ & $\begin{array}{l}12.28 \mathrm{a} \\
(+29.8)\end{array}$ & 71.45 c, d, e, f & 53.87 c, d, e & $816 \mathrm{~g}, \mathrm{~h}(-33)$ & 63.57 b, c & 5 \\
\hline T6 (CA) & $\begin{array}{l}28.30 \mathrm{~b} \\
(+11.6)\end{array}$ & $9.99 \mathrm{~b}(+15.9)$ & 65.6 h $(-13.7)$ & $54.24 \mathrm{c}, \mathrm{d}$ & 1306 b, c, d & $\begin{array}{l}57.41 \mathrm{e}, \mathrm{f} \\
(-9.3)\end{array}$ & 5 \\
\hline $\mathrm{T} 7(\mathrm{Mn}+\mathrm{CA})$ & 24.24 d, e & 9.15 b, c & $\begin{array}{l}69.60 \text { e, f, g, h } \\
(-8.4)\end{array}$ & $51.47 \mathrm{~d}, \mathrm{e}$ & $\begin{array}{c}1366 \text { b, c } \\
(+13)\end{array}$ & $\begin{array}{c}59.24 \mathrm{e}, \mathrm{f} \\
(-6.4)\end{array}$ & 5 \\
\hline $\mathrm{T} 8(\mathrm{Cu}+\mathrm{CA})$ & 26.56 b, c, d & 9.18 b, c & $\begin{array}{c}66.55 \mathrm{~g}, \mathrm{~h} \\
(-12.4)\end{array}$ & $54.31 \mathrm{c}, \mathrm{d}$ & 1498 a (+24) & $\begin{array}{l}58.23 \text { e, f } \\
(-8.0)\end{array}$ & 3 \\
\hline T9 (Zn + CA) & $\begin{array}{l}28.22 \mathrm{~b} \\
(+11.3)\end{array}$ & $9.13 \mathrm{~b}, \mathrm{c}$ & $\begin{array}{l}71.2 \mathrm{~d}, \mathrm{e}, \mathrm{f} \\
(-6.3)\end{array}$ & 54.43 c, d & $1133 \mathrm{f}$ & 60.03 c, d, e, f & 3 \\
\hline $\mathrm{T} 10(\mathrm{Mo}+\mathrm{CA})$ & 25.74 b, c, d & 12.32 a $(+43)$ & 73.38 c, d, e & 47.6 e $(-12.7)$ & 1229 d, e, f & 59.47 d, e, f & 5 \\
\hline T11 (B + CA) & $\begin{array}{c}22.26 \mathrm{e}, \mathrm{f} \\
(-12.4)\end{array}$ & 9.55 b, c & $\begin{array}{c}70.33 \mathrm{e}, \mathrm{f}, \mathrm{g} \\
(-7.4)\end{array}$ & $56.71 \mathrm{c}, \mathrm{d}$ & 1168 e $f$ & 60.82 c, d, e, f & 3 \\
\hline
\end{tabular}

*Means given within a column with the same letters are not significantly different at $\mathrm{P}<0.05$. Four replicates were used. The numbers in the brackets are increase (+) or decrease (-) from the control for a given seed nutrient constituent. Source of micro-nutrient compounds used were $\mathrm{MnCl}_{2}$, CuCl $\mathrm{C}_{2}$, $\mathrm{ZnCl}_{2}, \mathrm{MoO}_{3}$, and $\mathrm{H}_{3} \mathrm{BO}_{3} . \mathrm{C}=$ Control; $\mathrm{T}=$ Treatments. ${ }^{* *}$ Mo content was to low $(1-5 \mathrm{ppm})$ to be compared by SAS for significance value.

Table 4. Seeds macro-nutrient concentrations (\%) in soybeans as affected by soil micro-nutrient application and chelating agent citric acid (CA).

\begin{tabular}{|c|c|c|c|c|c|c|}
\hline Treaments & $\mathrm{Ca}$ & $\mathrm{K}$ & $\mathrm{Mg}$ & $\mathrm{P}$ & $\mathrm{N}$ & $\mathrm{S}$ \\
\hline $\mathrm{C}$ & $0.31 \mathrm{a}, \mathrm{b}, \mathrm{c}$ & $2.02 \mathrm{~b}, \mathrm{c}$ & $0.25 \mathrm{e}, \mathrm{f}$ & $0.79 \mathrm{c}, \mathrm{d}, \mathrm{e}$ & 5.77 b, c & $0.34 \mathrm{f}$ \\
\hline T1 (Mn) & $0.30 \mathrm{a}, \mathrm{b}, \mathrm{c}, \mathrm{d}$ & $2.02 \mathrm{~b}, \mathrm{c}$ & 0.26 c, d, e & 0.78 c, d, e & $5.88 \mathrm{~b}$ & 0.35 c, d, e, f \\
\hline $\mathrm{T} 2(\mathrm{Cu})$ & $0.31 \mathrm{a}, \mathrm{b}, \mathrm{c}, \mathrm{d}$ & $2.06 \mathrm{~b}, \mathrm{c}$ & $0.27 \mathrm{~b}, \mathrm{c}(+8.0)$ & 0.87 a $(+10.1)$ & $6.22 \mathrm{a}(+7.8)$ & 0.38 a (+11.8) \\
\hline T3 (Zn) & $0.33 \mathrm{a}$ & $2.03 \mathrm{~b}, \mathrm{c}$ & $0.25 \mathrm{e}, \mathrm{f}$ & $0.82 \mathrm{a}, \mathrm{b}, \mathrm{c}$ & $5.87 \mathrm{~b}$ & $0.37 \mathrm{a}, \mathrm{b}(+8.8)$ \\
\hline T4 (Mo) & 0.33 a & $2.1 \mathrm{a}, \mathrm{b}$ & $\begin{array}{c}0.27 \text { b, c, d } \\
(+8.0)\end{array}$ & $0.81 \mathrm{a}, \mathrm{b}, \mathrm{c}$ & 5.57 c, d, e & $\begin{array}{c}0.36 \text { b, c, d } \\
(+5.9)\end{array}$ \\
\hline T5 (B) & 0.29 c, d, e & $2.17 \mathrm{a}(+7.4)$ & 0.29 a $(+16.0)$ & $0.83 \mathrm{a}, \mathrm{b}, \mathrm{c}$ & $6.25 a(+8.3)$ & 0.38 a $(+11.8)$ \\
\hline T6 (CA) & $0.32 \mathrm{a}, \mathrm{b}, \mathrm{c}$ & 2.18 a (+7.9) & $0.28 \mathrm{~b}(+12.0)$ & $0.86 \mathrm{a}, \mathrm{b}(+8.9)$ & $5.48 \mathrm{~d}$, e $(-5.0)$ & $\begin{array}{c}0.36 \text { b, c, d, e } \\
\quad(+5.9)\end{array}$ \\
\hline $\mathrm{T} 7(\mathrm{Mn}+\mathrm{CA})$ & 0.30 b, c, d, e & $2.05 \mathrm{~b}, \mathrm{c}$ & $0.26 \mathrm{c}, \mathrm{d}, \mathrm{e}$ & 0.86 a (+8.9) & 5.78 c, d, e & 0.35 c, d, e, f \\
\hline $\mathrm{T} 8(\mathrm{Cu}+\mathrm{CA})$ & 0.29 c, d, e & $2.05 \mathrm{~b}, \mathrm{c}$ & $0.26 \mathrm{~d}, \mathrm{e}$ & $0.85 \mathrm{a}, \mathrm{b}(+7.6)$ & 5.62 b, c, d, e & $0.36 \mathrm{~b}, \mathrm{c}(+5.9)$ \\
\hline T9 (Zn + CA) & 0.28 e $(-9.7)$ & $2.05 \mathrm{~b}, \mathrm{c}$ & 0.25 e, f, g & 0.86 a (+8.9) & 5.78 b, c & $0.36 \mathrm{~b}, \mathrm{c}(+5.9)$ \\
\hline $\mathrm{T} 10(\mathrm{Mo}+\mathrm{CA})$ & $0.32 \mathrm{a}, \mathrm{b}, \mathrm{c}$ & $1.88 \mathrm{~d}(-6.9)$ & $0.24 \mathrm{f}, \mathrm{g}, \mathrm{h}$ & $0.74 \mathrm{e}$ & 5.73 b, c, d & 0.35 e, f \\
\hline $\mathrm{T} 11$ (B + CA) & $0.28 \mathrm{~d}$, e $(-9.7)$ & $1.96 \mathrm{c}, \mathrm{d}$ & $0.23 \mathrm{~g}, \mathrm{~h}(-8.0)$ & 0.80 b, c, d & 5.72 b, c, d & $0.35 \mathrm{~d}, \mathrm{e}, \mathrm{f}$ \\
\hline
\end{tabular}

${ }^{*}$ Means given within a column with the same letters are not significantly different at $\mathrm{P}<0.05$. Four replicates were used. The numbers in the brackets are $\%$ increase $(+)$ or decrease $(-)$ from the control. Source of micro-nutrient compounds used were $\mathrm{MnCl}_{2}, \mathrm{CuCl}_{2}, \mathrm{ZnCl}_{2}, \mathrm{MoO}_{3}$, and $\mathrm{H}_{3} \mathrm{BO}_{3} . \mathrm{C}=$ Control; $\mathrm{T}=$ Treatments. 
$\mathrm{H}_{3} \mathrm{BO}_{3}$ decreased Na concentrations in seeds by $39 \%$, 25\%, and 33\%, respectively, but the same applications had increased Na concentrations in leaves by $211 \%, 141 \%$, and 71\%, respectively (Table 1 and Table 3). Application of CA increased B and $\mathrm{Cu}$ in seeds by $11.6 \%$ and $15.9 \%$, respectively. Combination application of $\mathrm{Cu}+\mathrm{CA}$ increased Na concentration by $24 \%$.

Application Zn + CA increased B by 11.3\%. Application of Mo + CA increased Cu in seeds by 43\% (Table 3). Application of chealting agent CA decreased $\mathrm{Fe}$ and $\mathrm{Zn}$ in seeds by $13.7 \%$ and $9.3 \%$, respectively. Combining $\mathrm{Mn}+\mathrm{CA}$ also decreased $\mathrm{Fe}$ and $\mathrm{Zn}$ concentrations in seeds by $8.4 \%$ and $6.4 \%$. Similar trends were observed with the combination application of $\mathrm{Cu}+\mathrm{CA}$, which decreased Fe and $\mathrm{Zn}$ by 12.4 and 8.0, respectively. Mo + CA treatment decreased Mn by $12.7 \%$. B + CA treatment decreased B and Fe concentrations in seeds by $12.4 \%$ and 7.4\%, respectively. For micro-nutrients, usual trend was, $\mathrm{Na}>\mathrm{Fe}>\mathrm{Zn}>\mathrm{Mn}>\mathrm{B}>\mathrm{Cu}>\mathrm{Mo}$, agreeing with previous report [12]. Application of Mo in present experiment did result in a new trend, which was $\mathrm{Na}>\mathrm{Mo}>$ $\mathrm{Fe}>\mathrm{Zn}>\mathrm{Mn}>\mathrm{B}>\mathrm{Cu}$.

The treatments of $\mathrm{Cu}, \mathrm{Zn}, \mathrm{Mo}, \mathrm{B}$ and $\mathrm{CA}$ also increased macro-nutrients in the seeds (Table 4). Cu treatment increased Mg, P, $\mathrm{N}$ and S by $8.0 \%, 10.1 \%, 7.8 \%$ and $11.8 \%$, respectively. $\mathrm{Zn}$ application increased S by $8.8 \%$, and Mo application increased $\mathrm{Mg}$ and $\mathrm{S}$ by $8.0 \%$ and $5.9 \%$. B application increased $\mathrm{K}, \mathrm{Mg}, \mathrm{N}$ and $\mathrm{S}$ in seeds by 7.4\%, 16.0\%, 8.3\% and $11.8 \%$, respectively. CA application increased $\mathrm{K}, \mathrm{Mg}, \mathrm{P}$ and S by 7.4, 16.0, 8.9 and $5.9 \%$, respectively. Most of these treatments had positive impact on the accumulation of macro-nutrients in the soybean seeds. Irrespective of the treatments, the trend in macro-mineral concentration in seeds was $\mathrm{N}>\mathrm{K}>\mathrm{P}>$ $\mathrm{S}>\mathrm{Ca}>\mathrm{Mg}$, showing the same pattern as previously observed [12].

\section{Discussion}

Our present study demonstrates that there is direct and indirect effect of mineral treatments in increasing the minerals applied asvwell as affecting other minerals in the leaves and seeds except for $\mathrm{MnCl}_{2}$. $\mathrm{Mn}, \mathrm{Cu}, \mathrm{Zn}, \mathrm{Mo}$, $\mathrm{B}$, in combination treatment with chelating agent also had synergistic effect on several micro and macro nutrients in leaves and seeds. Other studies have reported that foliar B application increased B in leaves and seed concentration [20]. Both Mo and Mo + CA applications increased Mo in leaves to 179 and $446 \mathrm{mg} / \mathrm{kg}$, but in seeds, the application of Mo application at R3 stage resulted in high Mo in seeds (202 mg/kg). However, combination application Mo + CA had no significant influence on Mo accumulation in seeds. The role of CA in Mo + CA application in inhibiting the Mo accumulation in seeds during pod-filling stage is not clear. Recently, it is reported that, it is possible to produce Mo-rich seeds of several soybean cultivars by two foliar sprays of $400 \mathrm{~g}$ Mo ha ${ }^{-1}$, between the R3 and R5 stages, with a minimum interval of 10 days between sprays [21]. As a result of this method, considerable increases in seed Mo content were obtained, as much as $3000 \%$, in comparison to seeds obtained from plants which received no Mo, and this was not expected. Their goal was to produce plants with increased $\mathrm{N}$ and Mo contents in the grain, and higher grain yields. They have reported that in most cases, Mo-rich soybean seeds did not require any further application of Mo-fertilizer [21]. Therefore, Mo application may lead to an expected higher accumulation of Mo in seeds. The Mo application needs further investigation before conclusive recommendations are made.

Other studies were conducted in the fields where $\mathrm{Zn}, \mathrm{Mn}$, and Fe were applied to soybean. These studies showed similar effect of $\mathrm{Zn}, \mathrm{Mn}$, and Fe increase in different parts of plant tissues (roots, stems, leaves, and seeds) [22]-[25]. Zn, Fe, and Mn were partitioned in different parts of the plant tissues. It is reported in $\mathrm{Zn}$ application, Zn was observed more in pods. Zn application decreased Fe in seeds. Fe application increased Fe in seeds. In our previous research, we had seen that $\mathrm{FeCl}_{2}$ application increased $\mathrm{Fe}$ and $\mathrm{Zn}$ in seeds by $14.0 \%$ and $\mathrm{Cu}$ by $7.0 \%$ [12], and significant increase in $\mathrm{Cu}, \mathrm{B}$, and $\mathrm{Zn}$ concentrations in the seeds were also reported when the chelating agents DSEDTA, CA, and salicylic acid (SA) were applied [12].

In present study, CA alone or in combination applications $(\mathrm{Mn}+\mathrm{CA}, \mathrm{Cu}+\mathrm{CA}, \mathrm{Zn}+\mathrm{CA}, \mathrm{B}+\mathrm{CA})$ resulted in a decrease of $\mathrm{Fe}$ concentration in seeds. Why combination applications decreased Fe in seeds is not well known, and further research is needed. One of the possibilities is that this may be due to the nature of Fe immobility or due to the inhibiting effects of CA. Another possibility is that Fe present in oxidized state $\left(\mathrm{Fe}^{+3}\right)$ has a high binding constant with chelating agent CA and hence its translocation to the plant tissue may be slowed down compared to the rest of the minerals which have low binding constant [26] [27]. Previous studies were conducted on the selective effect of iron, ferrous source $\left(\mathrm{FeCl}_{2}\right)$ and different chelating agents on macro-nutrients of soybean seeds in previous studies [12], which showed that $\mathrm{Fe}^{+2}$ application increased $\mathrm{Ca}$, Mg, and $\mathrm{N}$ by $25.0 \%$, 
7.0\% and 5.5\%, respectively. Chelating agent $\mathrm{Fe}+\mathrm{CA}$ increased $\mathrm{K}$, Mg and $\mathrm{N}$ by $23.0 \%, 7.0 \%$ and $6.0 \%$, respectively.

\section{Conclusion}

In the current study and under greenhouse conditions, the soil applications of micro-nutrients $(\mathrm{Mn}, \mathrm{Cu}, \mathrm{Zn}, \mathrm{Mo}$ and B) individually or in combination with the chelating agent citric acid (AC) altered leaf and seed micro- and macro-nutrients, resulting in increasing $\mathrm{Cu}, \mathrm{Zn}, \mathrm{Mo}, \mathrm{B}, \mathrm{K}, \mathrm{N}, \mathrm{P}, \mathrm{Mg}$ and $\mathrm{S}$. The unexpected increase of Mo resulted from soil Mo application. Mo increase needs further studies. Since the current research is conducted under greenhouse conditions, the alteration of leaf or seed nutrients, due to the nutrient applications, are not conclusive unless further research is conducted under field conditions.

\section{Acknowledgements}

We would like to thank Ms. Sandra Mosley for soybean seed chemical analyses. We would like to thank Dr. Nacer Bellaloui, one of the co-authors, for supporting us with lab facilities and SAS analysis at USDA-ARS, Crop Genetics Research Unit, Stoneville, MS. First author would like to thank his daughter Neeta M. Goli for sharing her critics with the author. Her critics were valuable. This research was funded by Title III Part B.

\section{References}

[1] Ash, M. (2012) Soybean and Oilseeds. United States Department of Agriculture Economic Research Service. http://www.ers.usda.gov/topics/crops/soybeans-oil-crops.aspx

[2] Taylor, R.D. and Won, W.K. (2013) 2013 Outlook of the U.S. and World Corn and Soybean Industries, 2012-2022. Agribusiness \& Applied Economics Report No. 713, 1-27. http://ageconsearch.umn.edu/bitstream/157670/2/AAE713.pdf

[3] Messina, M. and Messina, V. (2010) The Role of Soy in Vegetarian Diets. Nutrients, 2, 855-888. http://dx.doi.org/10.3390/nu2080855 www.mdpi.com/journal/nutrients

[4] SoyaTech Growing Opportunities (2014) Soybeans and Oilseeds. http://www.soyatech.com/soy_oilseed_facts.htm

[5] Sakthivelu, G., Devi, M.K.A., Giridhar, P., Rajasekaran, T., Ravishankar, G.A., Nikolova, M.T., Angelov, G.B., Todorova, R.M. and Kosturkova, G.P. (2008) Isoflavone Composition, Phenol Content, and Antioxidant Activity of Soybean Seeds from India and Bulgaria. Journal of Agricultural and Food Chemistry, 56, 2090-2095. http://dx.doi.org/10.1021/jf072939a

[6] Potter, S.M., Baum, J.A., Teng, H.Y., Stillman, R.J., Shay, N.F. and Erdman, J.W. (1998) Soy Protein and Isoflavones: Their Effects on Blood Lipids and Bone Density in Post-Menopausal Women. American Journal of Clinical Nutrition, 68, 1375-1379.

[7] Messina, M.J. (1999) Legumes and Soybeans: Overview of Their Nutritional Profiles and Health Effects. The American Journal of Clinical Nutrition, 70, 439-450. http://ajcn.nutrition.org/content/70/3/439s.full

[8] Wan, J., Liu, C.M., Liu, W., Tu, Z.C., Li, C. and Zhang, W.Q. (2010) Determination of Mineral Elements in Soybean from Different Producing Areas by ICP-AES. Guang Pu Xue Yu Guang Pu Fen Xi, 30, 543-555. (In Chinese) http://www.ncbi.nlm.nih.gov/pubmed/20384163

[9] Michelfelder, A.J. (2009) Soy: A Complete Source of Protein. American Family Physician, 79, 43-47. http://www.ncbi.nlm.nih.gov/pubmed/19145965

[10] Tulchinsky, T.H. (2010) Micronutrient Deficiency Conditions: Global Health Issues. Public Reviews, 32, $243-255$. http://www.publichealthreviews.eu/show/f/28

[11] Xu, Y.F., Diaoguo, An, D.G., Li, H.J. and Xu, H.X. (2011) Review: Breeding Wheat for Enhanced Micronutrients. Canadian Journal of Plant Sciences, 91, 231-237. http://dx.doi.org/10.4141/CJPS10117

[12] Goli, M.B., Pande, M. and Bellaloui, N. (2012) Effects of Chelating Agents on Protein, Oil, Fatty Acids, and Minerals in Soybean Seed. Agricultural Sciences, 3, 517-523. http://dx.doi.org/10.4236/as.2012.34061 http://www.scirp.org/journal/PaperInformation.aspx?paperID=20174\&publishStatus=2\#.VIY2e2c6U1I

[13] Vahedi, A. (2010) Study of the Effects of Micronutrient Application on the Absorption of Macro- and Micronutrients in the Soybean Cultivar Telar in the North of Iran. Journal of American Science, 7, 1252-1257. http://www.jofamericanscience.org/journals/am-sci/am0706/189_5747am0706_1252_1257.pdf

[14] Yasari, E. and Vahedi, A. (2012) Micronutrients Impact on Soybean (Glycine max (Merrill)) Qualitative and Quantitative Traits. International Journal of Biology, 4, 112-118. http://dx.doi.org/10.5539/ijb.v4n2p112 
http://www.ccsenet.org/journal/index.php/ijb/article/view/10561/10838

[15] Simons, J.N., Swindler, R. and Benedict, H.M. (1961) Absorption of Chelated Iron by Soybean Roots in Nutrient Solutions. Physiology, 37,460-466. http://dx.doi.org/10.1104/pp.37.4.460 http://www.ncbi.nlm.nih.gov/pmc/articles/PMC549816/pdf/plntphys00427-0008pdf

[16] Whitaker, J., Harris, G., Kemerait, R., Prostko, E., Roberts, P., Smith, N., Smith, A. and Sumner, P. (2015) Georgia Soybean Production Guide. Printing Made Possible by Georgia Agricultural Commodity Commission for Soybeans. http://www.caes.uga.edu/commodities/fieldcrops/soybeans/documents/2015GeorgiaSoybeanProductionGuide-website. pdf

[17] Environmental Protection Agency(EPA), USA Method (2007) Microwave Assisted Acid Digestion of Sediments, Sludges, Soils and Oil. http://www3.epa.gov/epawaste/hazard/testmethods/sw846/pdfs/3051a.pdf

[18] SAS (2001) SAS 9.1 TS Level 1M3, Windows Version. 5.1.2600. SAS Institute, Cary.

[19] Stanton, M., Spann, T., Pande, M. and Goli, M. (2011) Qualitative and Quantitative Distribution of Trace Elements Found in the Leaves of the Soybean Plants that Were Exposed to Chelating Agents and Ferrous Ion. Mississippi Academy of Sciences Meeting, 75, 31. http://msacad.org/journal/jan11journal/mas11a.pdf

[20] Bellaloui, N., Hu, Y., Mengistu, A., Kassem, M.A. and Abel, C.A. (2013) Effects of Foliar Boron Application on Seed Composition, Cell Wall Boron, and Seed $\delta(15) \mathrm{N}$ and $\delta(13) \mathrm{C}$ Isotopes in Water-Stressed Soybean Plants. Frontiers in Plant Science, 23, 270. http://www.ncbi.nlm.nih.gov/pubmed/23888163

[21] Rubens, J.C. and Ricardo, S.A. (2009) Molybdenum-Enriched Soybean Seeds Enhance N Accumulation, Seed Yield and Protein Content in Brazil. Field Crops Research, 3, 219-224. http://www.sciencedirect.com/science/article/pii/S0378429008001846

[22] Soheil, K., Ghorban, N.M., Hosein, H.S.A., Farokh, D.K. and Babak, D. (2013) Micronutrients Distribution in Soybean Plant with Zn, Fe, and Mn Application. Annual Review \& Research in Biology, 3, 83-91. http://webcache.googleusercontent.com/search?q=cache:RW0XAIvAg-oJ:sciencedomain.org/download/MTA0MkBA $\underline{\mathrm{cGY}+\& \mathrm{~cd}=1 \& \mathrm{hl}=\mathrm{en} \& \mathrm{ct}=\mathrm{clnk} \& \mathrm{gl}=\mathrm{us}}$

[23] Jha, A.N. and Chandel, A.S. (1987) Response of Soybean to Zinc Application. Indian Journal of Agronomy, 32, 354358.

[24] Rhoads, F.M. (1984) Soybean Response to Zinc Fertilization. Soil and Crop Science Society Florida Proceeding, 43, 46-48.

[25] Boote, K.J., Gallaher, R.N., Robertson, W.K., Hinson, K. and Hammond, L.C. (1980) Effects of Foliar Fertilization on Photosynthesis, Leaf Nutrition, and Yield of Soybean. Agronomy Journal, 72, 271-275.

[26] Brown, J.C., Tiffin, L.O. and Holmes, R.S. (1960) Competition between Chelating Agents and Roots as Factor Affecting Absorption of Iron and Other Ions by Plant Species. Plant Physiology, 35, 878-886. http://dx.doi.org/10.1104/pp.35.6.878

[27] Eby, G. (2006) Stability Constants (log K1) of Various Metal Chelates. In: Furia, T.E., Ed., CRC Handbook of Food Additives, Chapter 6: Sequestrants in Foods. http://www.coldcure.com/html/stability_constants.html 